\title{
A state with negative binding energy induced by coherent transport in a quantum wire
}

\author{
Vidar Gudmundsson, ${ }^{1}$ Chi-Shung Tang, ${ }^{2}$ and Andrei Manolescu ${ }^{1}$ \\ ${ }^{1}$ Science Institute, University of Iceland, Dunhaga 3, IS-107 Reykjavik, Iceland \\ ${ }^{2}$ Physics Division, National Center for Theoretical Sciences, P.O. Box 2-131, Hsinchu 30013, Taiwan
}

\begin{abstract}
In a two-dimensional quantum wire in a perpendicular magnetic field with a smooth embedded repulsive scattering potential we find in the multimode conductance resonances caused by bound states with negative binding energy. These resonances are the counterexamples to well known dips in the conductance and evanescent states caused by quasi-bound states of attractive scattering centers in the wire.
\end{abstract}

PACS numbers: 73.21.Hb, 73.22.Dj, 73.63.Nm

Coherent quantum transport of electrons through quantum wires has been measured and modeled by many groups during the last one and half decade. The conductance quantization and deviations from it have been a focus of many researchers. Early on, it was discovered that an attractive scatterer can block a conduction channel totally for a narrow range of energy just at the end of a conduction plateau or step $\stackrel{1,2}{ }$ This sharp dip structure was analyzed to be caused by a total reflection of the incoming electron wave in one channel due to a quasibound state originating from the evanescent mode in the next higher energy subband.

Without an external constant magnetic field nothing corresponding to this blocking can occur in a quantum wire with an embedded repulsive scatterer as it has no quasi-bound states in contrast to the attractive scatterer. In an external magnetic field this argument does not hold as can be inferred by a publication of Laughlin. ${ }^{3}$

In this publication we will show that, indeed, the transport properties of a quantum wire with an embedded repulsive scatterer in a constant external magnetic field can exhibit signs of quasi-bound states. We will demonstrate that these states can reveal their presence both by resonance transmission peaks and by dips in the conductance indicating resonant backscattering processes.

We consider a multi-mode transport of electrons along the $x$-direction through a two-dimensional quantum wire defined by a parabolic confinement in the $y$-direction with the characteristic energy $E_{0}=\hbar \Omega_{0}$. The electrons incident from the left $(x \rightarrow-\infty)$ impinge on a smooth Gaussian scattering potential $V_{s c}=V_{0} \exp \left(-\beta r^{2}\right)$ Together the magnetic field $\mathbf{B}=B \hat{\mathbf{z}}$ and the parabolic confinement define a natural length scale $a_{w}=\sqrt{\hbar /\left(m^{*}\right) \Omega_{w}}$, where $\Omega_{w}=\sqrt{\omega_{c}^{2}+\Omega_{0}^{2}}$, with the cyclotron frequency $\omega_{c}=e B /\left(m^{*} c\right)$, is the natural frequency of the quantum wire in a magnetic field. The length $a_{w}$ can be considered as an effective magnetic length in the wire system. A mixed momentum-coordinate presentation of the wave functions $\Psi_{E}(p, y)=\int d x \psi_{E}(x, y) e^{-i p x}$ and expansion in channel modes $\Psi_{E}(p, y)=\sum_{n} \varphi_{n}(p) \phi_{n}(p, y)$ in terms of the eigenfunctions for the pure parabolically confined wire in magnetic field leads to a coupled set of Lippman-Schwinger integral equations in momentum space. These equations are then transformed into integral equations for the $T$-matrix in order to facilitate numerical evaluation. ${ }^{4.5}$ The conductance is evaluated according to the Landauer-Büttiker formalism together with the scattering wave functions from the $T$-matrix.

We consider a broad parabolic quantum wire with confinement energy $\hbar \Omega_{0}=1 \mathrm{meV}$. At a vanishing magnetic field $B=0$ this energy corresponds to $a_{w}=33.7 \mathrm{~nm}$. We select a fairly narrow but smooth scatterer in the center of the wire with $\beta=1 \times 10^{-2} \mathrm{~nm}^{-2}$, equivalent to $\beta a_{w}^{2}=11.4$ at $B=0$, or in other words the scattering potential has reached $e^{-1}$ at $r \approx 10 \mathrm{~nm}$, close to the value of the effective Bohr radius in GaAs $a_{0}^{*}=9.79$ $\mathrm{nm}$. In the numerical calculations we have included at least 13 channel modes in the wire and use an unevenly spaced grid in momentum space to apply a repeated 4point Gaussian integration to the integral equations for the T-matrix. Numerical accuracy is assured through comparison to solutions obtained with a larger basis set.

In order to understand better the results for a wire with a repulsive scatterer we first show in Fig. 11 results for a wire with an attractive scatterer. To compare conductance curves for different values of $B$ we show them as functions of $X=E /\left(\hbar \Omega_{w}\right)+1 / 2$. The integer part of the parameter $X$ counts how many channels in the wire are open for transport for an incoming electron with propagating energy $E$. At low magnetic field, $B=0.5 \mathrm{~T}$, dips are seen in the conductance curve just before $X$ assumes integer values. These well known dips can in a perturbative picture be explained as being caused by a backscattering of the incoming electron in subband $n$ by an evanescent state in subband $n+1$ that the scattering potential has lowered into the gap between the subbands just below the $n+1$ subband. The probability density of the electronic state in the first subband with $X=1.996$ is shown in Fig 1(b). On the incident side, the left side, we see the interference pattern of the incoming and outgoing $n=1$ state, and on the right side there is only the evanescent probability.

For a higher magnetic field, $B=3 \mathrm{~T}$, the Lorentz force corresponding to the kinetic energy necessary to reach the evanescent state is large enough to press the electron into one side of the wire far enough from its center 

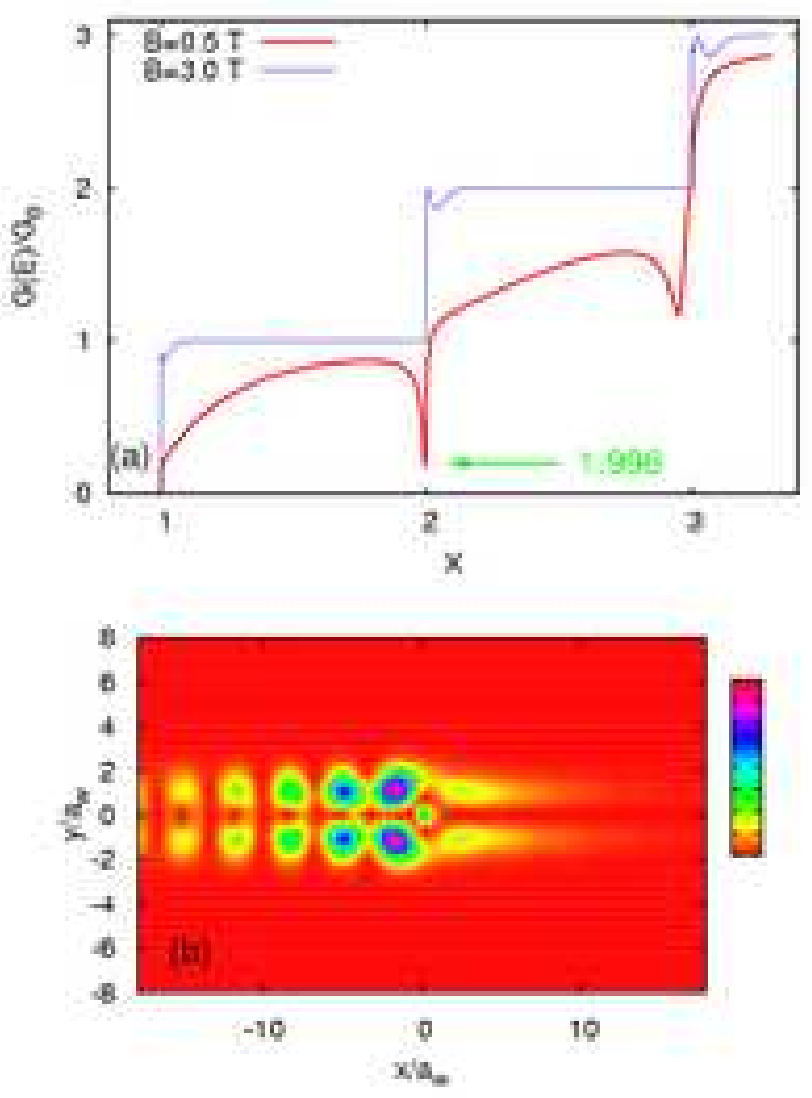

FIG. 1: (Color online) (a) The conductance in units of $G_{0}=$ $2 e^{2} / h$ of a broad wire with one embedded narrow potential well at the center $x=0$, as function of $X=E /\left(\hbar \Omega_{w}\right)+1 / 2$. (b) The probability density of the scattering state corresponding to an incident state with $X=1.996$ and $n=1$. $E_{0}=\hbar \Omega_{0}=1.0 \mathrm{meV}, V_{0}=-8 \mathrm{meV}, \beta=10^{-2} \mathrm{~nm}^{-2}$, and for GaAs $a_{0}=9.79 \mathrm{~nm}$. $a_{w}=29.34 \mathrm{~nm}$ at $B=0.5 \mathrm{~T}$, and $a_{w}=14.68 \mathrm{~nm}$ at $B=3.0 \mathrm{~T}$.

suppressing the overlap between the incoming and the evanescent state. As a result there is no dip found, and the only clear deviation from perfect conductance is in the beginning of each conductance step where the Lorentz force is small enough to still allow for an encounter between the electron and the scattering potential.

This together with the curious fact noted by Laughlin ${ }^{3}$ that two electron restricted to a plane perpendicular to a constant magnetic field can form a bound state with negative binding energy leads us to the following train of thought: If two electrons form a bound state, so can also one do around a smooth potential hill. The potential hill in the middle of a quantum wire will lift at least one state higher into the band where it originated. This quasi-bound state just above the band minimum might influence the scattering at high magnetic field just in the beginning of a new conductance step. In a flat two-dimensional system the state would be a true bound state, but in a quantum wire there is always an equipo- tential line along the wire edge with the same energy as the bound state guaranteeing at least a vanishingly small overlap between the edge and the bound state.

The parameters chosen earlier in this paper for the wire and the scattering potential proved fruitful in the search for a bound state of an electron around a hill. In Fig. 2(a) we show the conductance of a wire with an embedded potential hill, a repulsive scatterer. A fine structure is visible at the beginning of each conductance step. In Fig. 2(b) we focus in on the beginning of the second step for two close values of $B$. The arrows point


FIG. 2: (Color online) The conductance in units of $G_{0}=$ $2 e^{2} / h$ of a broad wire with one embedded narrow potential hill at the center $x=0$, as function of $X=E /\left(\hbar \Omega_{w}\right)+1 / 2$ (a), and the same repeated for a narrow range of $X$. $E_{0}=$ $\hbar \Omega_{0}=1.0 \mathrm{meV}, V_{0}=+8 \mathrm{meV}, \beta=10^{-2} \mathrm{~nm}^{-2}$.

at a transmission resonance at $X=2.054$ and a dip at $X=2.242$ for $B=3.0 \mathrm{~T}$. We shall also analyze a dip at the beginning of the first step at $X=1.242$ for an electron entering the wire in the $n=1$ channel mode at $B=2.0 \mathrm{~T}$. The corresponding probability densities for the electronic states are seen in Fig. B.

The kinetic energy of incoming electrons close to the transmission peak at $X=2.054$ is low leading to a small Lorentz force acting on them. The electrons thus encounter the scattering potential and there is some 

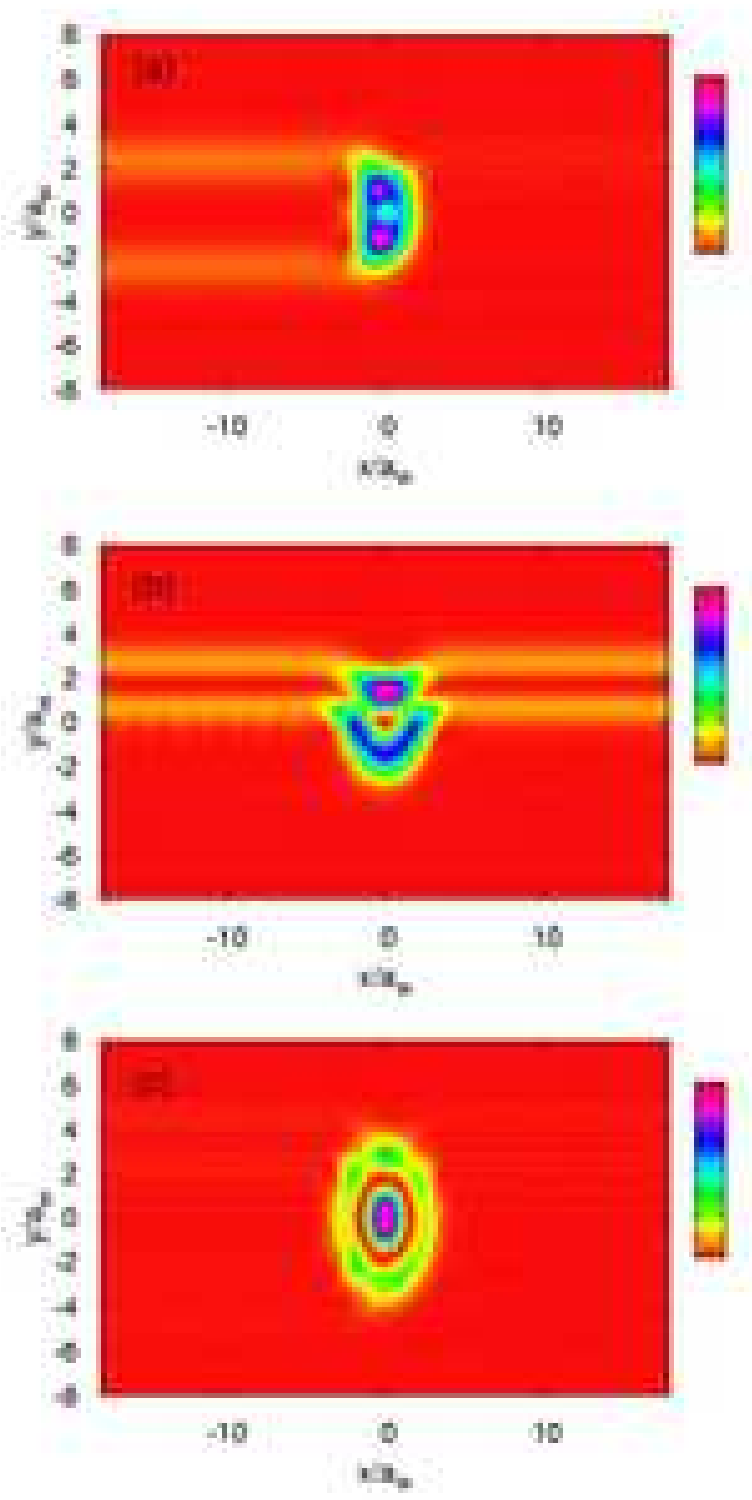

FIG. 3: (Color online) Corresponding to special values of $X$ in Fig. 2 the probability density of the scattering state corresponding to an incident state with $X=1.242, n=1$, and $B=2.0 \mathrm{~T}(\mathrm{a}), X=2.054, n=2$, and $B=3.0 \mathrm{~T}(\mathrm{~b})$, and $X=2.242, n=2$, and $B=3.0 \mathrm{~T}$ (c). $E_{0}=\hbar \Omega_{0}=1.0 \mathrm{meV}$, $V_{0}=+8 \mathrm{meV}, \beta=10^{-2} \mathrm{~nm}^{-2}$.

backscattering, though small. The probability density in Fig. 3(b) and the corresponding transmission peak in Fig. 2 shows us that at $X=2.054$ the electrons come into resonance with a quasi-bound state with binding energy of approximately $-0.29 \mathrm{meV}$. This low energy and the potential curvature of the wire give the probability density a shape that reflects both the structure of the resonant edge state and the circular scattering potential. The double hump structure of the incoming and the transmitted wave are signs of the $n=2$ mode or channel, the $n=1$ channel is further away from the center of the wire as is discussed below.

At higher kinetic energy for the incoming electrons the Lorentz force is large enough to allow most of them to bypass the potential resulting in almost perfect conductance, except for the dip at $X=2.242$ where the electrons come into resonance with a higher lying quasibound state that causes some of them to be backscattered. The probability density of this state is displayed in Fig. [3(c). It is long lived judging from the narrowness of the dip and the strength of the probability density that subdues almost the incoming and the reflected wave on the color scale used here. The binding energy of this state is $-1.18 \mathrm{meV}$ and the smaller coupling to the edge states makes its symmetry much closer to the circular symmetry of the scatterer. The double ring structure of this state suggests that there are more quasi-bound states with simpler structure and larger negative binding energy but their still smaller coupling to the edge states makes them invisible to our calculation.

A simple estimate of the binding energy of the scattering potential neglecting the confinement of the wire and using the semiclassical quantization condition of Bohr places the value of $-1.18 \mathrm{meV}$ in between values obtained by assuming the quantum numbers 2 and $2+1 / 2$. This is in accordance with the double hump structure we see in the probability density in Fig. 3(c).

It should be stated here that the probability densities for the $n=1$ states corresponding to the $n=2$ states in Fig. 3(b) and (c) at $B=3 \mathrm{~T}$ are straight edge states with their maxima at $y \approx 8 a_{w}$. So, we are observing a scatterer with radius approximately $0.8 a_{w}$, the bound state in Fig. [3 (b) has a radius of approximately $3 a_{w}$, and the distance from the bound state to the edge is approximately $5 a_{w}\left(a_{w}=14.7 \mathrm{~nm}\right.$ here). So, indeed, our scatterer is narrow but smooth leading to quasi-bound states of simple structure.

In Fig. 31 (a) is the probability density corresponding to to the dip at $X=1.242$ in the first subband. We can faintly see the incoming and the reflected waves. Here $B=2.0 \mathrm{~T}$ and due to the greater magnetic length or $a_{w}$ than for $B=3.0 \mathrm{~T}$ the symmetry of the quasi-bound state is influenced by the edge states. The resonance dip for this structure is deeper at $B=2.1 \mathrm{~T}$ (see Fig. 2(a)) but the faint probability for the incoming and the outgoing wave at $B=2.0$ T shows us the effective width of the wire here for this $n=1$ state.

The resonance at $B=3 \mathrm{~T}$ in Fig. 2(a) corresponding to the state in Fig. 3(c) is narrow reflecting a long lived state. At a lower magnetic field $B=2 \mathrm{~T}$ the corresponding resonance is broad indicating a relatively short lived resonance state producing it. Exactly this can be verified

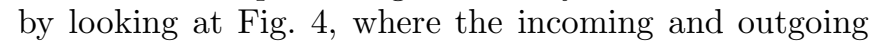
waves in the $n=2$ channel are clearly seen and one can infer from the structure of the probability density that semiclassically speaking the electrons undergoe few reflections before being returned. 


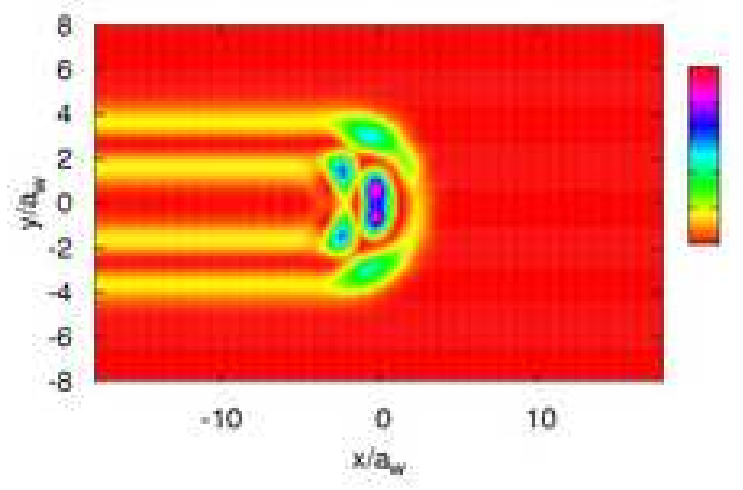

FIG. 4: (Color online) The probability density of the scattering state corresponding to an incident state with $X=2.29$, $n=2$, and $B=2.0 \mathrm{~T}$ corresponding to a broad resonance in Fig. 2] a) $E_{0}=\hbar \Omega_{0}=1.0 \mathrm{meV}, V_{0}=+8 \mathrm{meV}, \beta=10^{-2}$ $\mathrm{nm}^{-2}$.

We have demonstrated that quasi-bound states of a repulsive potential hill in a quantum wire in an external magnetic field can leave their fingerprints on the conductance of the system for suitably selected parameters. A small coupling to the edge states gives a long lived quasi-bound state with the symmetry of the scatterer, but the small coupling may hinder the state in affecting the conductance of the system. The narrow but smooth potential hill in an external magnetic field behaves like a "quantum peg" to which the electrons can be hooked for some time in contrast to the more familiar picture of the quasi-bound state of a quantum dot where one imagines the electron rattling for a short time in a bowl-like structure, the dot.

The use of a smooth scattering potential with feature lengths comparable to the effective Bohr radius in the system has allowed us to make visible the effects of fairly simple "low-lying" quasi-bound states at intermediate strengths of magnetic field $B \approx 3 \mathrm{~T}$. Clearly the effects of these quasi-bound states on the conductance are smaller than the effects of the corresponding states of an attractive scatterer so they are probably not easy to detect experimentally.

Here we have focused our view on the bound state of a smooth repulsive scatterer in a quantum wire in a perpendicular magnetic field as a complimentary system to the wire with an embedded attractive scatterer, and have compared the effects of these two scatterers on the conductance of the system. A similar, system has been considered analytically in the extreme quantum limit where a one band limit could be applied by Jain and Kivelson in order to explain the break down of the quantum Hall effect in narrow constrictions,$\underline{6}$ Takagaki and Ferry used a hard wall version of both a quantum strip and a scatterer to explore with a tight-binding formalism how a circulating edge channel around an antidot leads to an Aharonov-Bohm-type oscillation of the conductance as the magnetic field normal to the strip is varied ${ }^{7}$ They assume the diameter of the antidot is large compared to the characteristic wavelength of the electrons and focus their attention on the magnetic coupling of the the electron waves through the constriction on each side of the antidot. Corresponding experimental system with two large antidots has been investigated by Gould et al 8

We expect that a larger hard wall scatterer may also produce quasi-bound states (with edge state character around the scatterer) that could influence the conductance of the system, but we believe that those states might produce effects in the conductance that are harder to find due to their very low binding energy and closeness to the beginning of a conductance plateau.

\section{Acknowledgments}

The research was partly funded by the Research and Instruments Funds of the Icelandic State, the Research Fund of the University of Iceland, and the National Science Council of Taiwan. C.S.T. acknowledges the computational facility supported by the National Center for High-performance Computing of Taiwan.
1 P. F. Bagwell, Phys. Rev. B 41, 10354 (1990).

2 S. A. Gurvitz and Y. B. Levinson, Phys. Rev. B 47, 10578 (1993).

3 R. B. Laughlin, Phys. Rev. B 27, 3383 (1983).

4 S. A. Gurvitz, Phys. Rev. B 51, 7123 (1995).

${ }^{5}$ V. Gudmundsson, Y.-Y. Lin, C.-S. Tang, V. Moldoveanu, J. H. Bardarson, and A. Manolescu, Phys. Rev. B 71, in press (2005).
6 J. K. Jain and S. A. Kivelson, Phys. Rev. Lett. 60, 1542 (1988).

7 Y. Takagaki and D. K. Ferry, Phys. Rev. B 48, 8152 (1993).

8 C. Gould, A. S. Sachrajda, Y. Feng, A. Delage, P. J. Kelly, K. Leung, and P. T. Coleridge, Phys. Rev. B 51, 11213 (1995). 